This is the peer reviewed accepted manuscript of:

Protasoni, M., Bruno, C., Donati, M. A., Mohamoud, K., Severino, M., Allegri, A., Robinson, A., et al. (2020). Novel compound heterozygous pathogenic variants in nucleotide-binding protein like protein (NUBPL) cause leukoencephalopathy with multi-systemic involvement.. Molecular genetics and metabolism, 129 (1), 26-34.

Final version available at: https://doi.org/10.1016/i.ymgme.2019.11.003

Rights / License:

The terms and conditions for the reuse of this version of the manuscript are specified in the publishing policy. For all terms of use and more information see the publisher's website.

This item was downloaded from IRIS Università di Bologna (https://cris.unibo.it/)

When citing, please refer to the published version. 


\section{Novel compound heterozygous pathogenic variants in nucleotide-binding protein like protein (NUBPL) cause leukoencephalopathy with multi- systemic involvement.}

Margherita Protasoni1, Claudio Bruno2, Maria Alice Donati3, Khadra Mohamoud1, Mariasavina Severino4, Anna Allegri5, Alan J Robinson1, Aurelio Reyes1, Massimo Zeviani1,*, Caterina Garone1,6,*

1Medical Research Council Mitochondrial Biology Unit, University of Cambridge, Hills Road, CB20XY, Cambridge, UK

2 Center of Translational and Experimental Myology, IRCCS Giannina Gaslini Institute, via Gerolamo Gaslini 5, 16147, Genoa, Italy

3Metabolic Unit, A. Meyer Children's Hospital, viale Pieraccini 24, 50139, Florence, Italy

4Neuroradiology Unit, IRCCS Giannina Gaslini Institute, via Gerolamo Gaslini 5, 16147, Genoa, Italy

${ }_{5}$ Pediatric Clinic Unit, IRCCS Giannina Gaslini Institute, via Gerolamo Gaslini 5, 16147, Genoa, Italy

6 Dipartimento di Scienze Mediche e Chirurgiche, Centro di Ricerca Biomedica Applicata, Università di Bologna, via Massarenti, 11, 40100, Bologna

*Corresponding authors:

Caterina Garone, e-mail: caterina.garone@unibo.it

Massimo Zeviani, e-mail: mdz21@mrc-mbu.cam.ac.uk

Medical Research Council Mitochondrial Biology Unit, University of Cambridge, Wellcome Trust/MRC Building, Cambridge Biomedical Campus, Hills Road, Cambridge CB2 0XY, UK

Phone: $+44(0) 1223252750$

Fax: $+44(0) 1223252815$

GRANT NUMBERS

Core Grant from the MRC (Grant MC_UU_00015/5);

ERC Advanced under Grant FP7-322424 and NRJ-Institut de France Grant European

Commission under 'Marie Skłodowska-Curie Actions', Individual Fellowship-Reintegration

Panel (Mitobiopath-705560)(CG). 


\begin{abstract}
NUBPL (Nucleotide-binding protein like) protein encodes a member of the Mrp/NBP35 ATPbinding proteins family, deemed to be involved in mammalian complex I (CI) assembly process. Exome sequencing of a patient presenting with infantile-onset hepatopathy, renal tubular acidosis, developmental delay, short stature, leukoencephalopathy with minimal cerebellar involvement and multiple OXPHOS deficiencies revealed the presence of two novel pathogenic compound heterozygous variants in NUBPL (p.Phe242Leu/p.Leu104Pro). We investigated patient's and control immortalized fibroblasts and demonstrated that both the peripheral and the membrane arms of complex I are undetectable in mutant NUBPL cells, resulting in virtually absent $\mathrm{CI}$ holocomplex and loss of enzyme activity. In addition, complex III stability was moderately affected as well. Lentiviral-mediated expression of the wild-type NUBPL cDNA rescued both CI and CIII assembly defects, confirming the pathogenicity of the variants. In conclusion, this is the first report describing a complex multisystemic disorder due to NUBPL defect. In addition, we confirmed the role of NUBPL in Complex I assembly associated with secondary effect on Complex III stability and we demonstrated a defect of mtDNA-related translation which suggests a potential additional role of NUBPL in mtDNA expression.
\end{abstract}

Key words: Mitochondrial disorders, NUBPL, human complex I, complex I assembly, complex I assembly factors, brain MRI. 


\section{Introduction}

The primary function of mitochondria is to provide energy to the cells. This role is carried out by the oxidative phosphorylation (OXPHOS) pathway, composed of five multi-subunit complexes and two mobile electron carriers (ubiquinone and cytochrome c), embedded in the inner mitochondrial membrane (IMM). Complexes I to IV carry out respiration, i.e. the transport of electrons extracted from nutrients to molecular oxygen, that sustains the generation of a proton gradient across the membrane, exploited by complex $\mathrm{V}$ to phosphorylate ADP, converting it into ATP. Human complex I (CI) is an initial step of the electron transport chain. It is composed of 45 different subunits (Vinothkumar, Zhu, \& Hirst, 2014) organized in three structural domains: a membrane arm, or P module, and two peripheral domains, the $\mathrm{N}$ and the $\mathrm{Q}$ modules, protruding in the mitochondrial matrix. In the $\mathrm{N}$ module, Nicotinamide Dehydrogenase (NADH) is oxidised by flavin mononucleotide (FMN) and electrons are passed through a chain of 8 iron-sulfur (Fe-S) clusters, and eventually transferred to ubiquinone, bound to the Q module. For every oxidised NADH molecule, the P-module pumps four protons across the inner membrane from the matrix to the intermembrane space of mitochondria, contributing to the formation of the protonmotive force (Birrell, Morina, Bridges, Friedrich, \& Hirst, 2013; Mimaki, Wang, McKenzie, Thorburn, \& Ryan, 2012). Isolated mitochondrial CI deficiency (MIM\# 252010), due to pathogenic variants in CI subunits or assembly factors, causes severe decrease of energy production and is the most common cause of OXPHOS disorders (Nouws, Nijtmans, Smeitink, \& Vogel, 2012). Nucleotide-binding protein like (NUBPL) (MIM\# 613621) encodes a member of the Mrp/NBP35 ATP-binding proteins family (Bych et al., 2008; Sheftel et al., 2009). Similar to cytosolic members of this protein family, e.g. Cfd1 and Nbp35, NUBPL is able to bind Fe-S clusters thanks to the presence of two highly conserved cysteine residues in a CxxC motif in the $\mathrm{C}$-terminal domain of the protein, but the precise molecular mechanism remains unclear (Sheftel et al., 2009). NUBPL defect causes a leukoencephalopathy with a distinct brain MRI pattern predominantly involving cerebellar cortex, deep white matter and corpus callosum at early stage and progressing to severe cerebellar atrophy and brainstem involvement at the late stage (Kevelam et al., 2013). Currently, 10 patients with NUBPL pathogenic variants are reported in the literature (Balint et al., 2019; Calvo et al., 2010; Kevelam et al., 2013; Tenisch et al., 2012; Tucker et al., 2012) presenting a neurodevelopmental disorder with mild to severe ataxia, dystonia, spasticity and dysarthria (Table I-Figure1). Here, we report for the first time a complex multisystemic syndrome due to novel compound heterozygous pathogenic variants in NUBPL in a 13-yearold girl. Functional analyses were previously limited to the determination of CI enzymatic activity and steady-state levels in human defective NUBPL samples. In this study, we have performed an extensive biochemical, genetic and protein analysis of our patient-derived fibroblast cell line harbouring compound heterozygous NUBPL pathogenic single nucleotide variants bringing new insights into the disease mechanism of NUBPL.

\section{Case Report}

A 13-year-old girl, the first child of healthy unrelated parents, was born at term after uneventful pregnancy and normal delivery. Birth weight was 3,330 g. Perinatal period was normal (APGAR score 8/10). At age 18 months, she presented with failure to thrive (HP:0001508) and developmental delay (HP:0001263). At age 30 months, a type III renal tubular acidosis (HP:0001947) was identified. Laboratory investigations showed increased levels of lactate in plasma and in urine.

At this age neurological assessment documented generalized hypotonia (HP:0001290), wasting and weakness with positive Gowers sign (HP:0003198). Brain MRI revealed an asymmetric leukoencephalopathy (HP:0002352) with predominant fronto-parietal involvement, swelling of the corpus callosum and restricted diffusion at the edges of the affected white matter, 
associated with a small cortical cerebellar lesion (Figure 2A-E). Brain MR spectroscopy revealed lactate peaks at the level of both affected and normalappearing cerebral white matter (Figure 2K, L).

At 6 years, mild hepatomegaly (HP:0002240), short stature (HP:0004322) and severe osteoporosis (HP:0000939) were noted. Endocrinological evaluation detected a complete growth hormone deficiency (HP:0000824) and replacement treatment was started. At 7 years of age, pulmonary CT scan revealed interstitial lung disease (HP:0006530). Sweat test was negative. At this time, the child showed generalized muscle hypotrophy, hyposthenia and signs of peripheral reduced oxygenation with nail clubbing. Follow-up brain MRI showed improvement of the cerebral white matter abnormalities, with decrease in both white matter swelling and extent of the signal abnormalities, whereas the cerebellar abnormalities had slightly worsened (Figure 2F-J). Brain MR spectroscopy demonstrated absence of lactate peaks in the affected white matter (Figure 2M). Cardiac (including ECG and echocardiography), funduscopy, and hearing assessments were normal. Over the following years the clinical course remained stable with satisfactory electrolyte balance and metabolic compensation and stability of motor functions. At 12 years of age she presented a critical episode characterized by hypertonia to the limbs with loss of contact and tachypnea. EEG showed significant slowing in right frontal seat.

Laboratory results indicated the presence of increased plasma levels of alanine, lactic and butyric acid. A muscle biopsy performed at 36 months revealed an irregular pattern of Cytochrome $\mathrm{C}$ Oxidase (COX) activity. In addition, mitochondrial respiratory chain activities measured in muscle homogenate showed a significant reduction in CI+III (NADH-Cytochrome $\mathrm{C}$ reductase) and a marginal reduction in CII+III (Succinate cytochrome c reductase) (Table II). At her last examination, at 13 years of age, she complained of easy fatigability and she started enteral nutrition.

\section{Results}

Genetics

Exome sequencing of patient's blood DNA identified two pathogenic variants in NUBPL (NM_025152.3; NP_079428.2; \#MIM 613621): c.726C $>$ G, p.Phe242Leu, in exon 9, not previously reported; c.311T $>$ C, p.Leu104Pro, in exon 4, previously reported in compound heterozygosity with another pathogenic variant. Sanger's sequencing in the parents, proved that the pathogenic variants were allelic, the c. $726 \mathrm{C}>\mathrm{G}$ being transmitted by the heterozygous father and the c.311T $>\mathrm{C}$ by the heterozygous mother. Both parents are heathy carrier. The two pathogenic variants are localized in two different functional domains of the protein, and are both highly conserved in different species. ClustalW alignment of NUBPL and the human orthologues of cytosolic Fe/S proteins Cfd1/Nbp35 (NUBP1/NUBP2) (Figure S1) shows that the first pathogenic variant (p.Leu104Pro) localizes in the Walker B motif, essential for nucleotide binding and hydrolysis (Leipe, Koonin, \& Aravind, 2003). The second pathogenic variant (p.Phe242Leu) is in close proximity to the $\mathrm{CxxC}$ binding residues and may affect the ability of the protein to bind the Fe-S clusters or to form homodimers (Netz et al., 2012). MutationTaster and SIFT softwares predicted both pathogenic variants to be deleterious for protein function.

\section{Biochemistry}

We first analyzed the steady-state levels of respiratory chain subunits, particularly those forming the different CI submodules in immortalized patient-derived skin fibroblasts (Signes \& Fernandez-Vizarra, 2018). SDS-PAGE based Western blot (WB) immunodetection showed strong reduction of the steady-state level of CI subunits localized in both the peripheral and membrane arms, suggesting a deleterious effect of the NUBPL pathogenic variants on the 
whole enzyme (Figure 3A-S2). Steady-state levels of subunits of other respiratory chain complexes (Figure 3A) and of Aconitase 2 (ACO2) (Figure 4A), a mitochondrial matrix protein containing a $4 \mathrm{Fe}-4 \mathrm{~S}$ cluster involved in the conversion of Citrate to Isocitrate, did not show abnormalities. Likewise, the amount of the Fe-S containing structural components of CIII (UQCRFS1) was not affected. 1 st and $2^{\text {nd }}$ dimension (1D and 2D) BN-PAGE analysis of DDMtreated samples demonstrated a dramatic reduction of the fully assembled CI in patient's fibroblasts, whereas both CIII and CII, as well as CIV, holocomplexes were normal (Figures 3B and 3C). These results confirm the high specificity of NUBPL for the assembly/stability of CI subunits.

In order to study the effect of the two pathogenic variants in the formation of supercomplexes (SC), we analyzed mitochondria extracted from fibroblasts treated with $1 \%$ digitonin by $1 \mathrm{D}$ and 2D BN-PAGE WB (Figures 3D and 3E). This analysis showed a marked reorganization of the $\mathrm{SC}$ in the patient, including the loss of the respirasome $\left(\mathrm{CI}+\mathrm{CIII}_{2}+\mathrm{CIV}\right)$, with the maintenance of the $\mathrm{CIII}_{2}+\mathrm{CIV}$ species. However, WB analysis of 2D BN using UQCRFS1, the only CIII subunit containing an iron-sulfur cluster, and UQCRC1, a CIII core subunit, showed accumulation of CIII sub-assemblies in the patient's cells (Figure 3E). Interestingly, similar results, although less drastic, were obtained from the analysis of another human cell line defective for the complex I assembly factor ACAD9, including the decrease in the amount of SC (Figure S3). (Garone et al., 2013) Besides Complex I assembly, NUBPL has been deemed to play a role in mitochondrial translation in Arabidopsis thaliana (Wydro et al., 2013). We analyzed mitochondrial translation by $\left[{ }_{35} \mathrm{~S}\right]-\mathrm{L}$-methionine metabolic labelling, and found that the amount of mtDNA-related subunits was moderately decreased in mutant fibroblasts compared to controls (Figure 3F-S4), and also to the ACAD9 defective fibroblasts (Figure S5). Spectrophotometric analysis of the respiratory chain complexes confirmed severe CI deficiency with $22 \%$ residual activity of patient's vs controls' fibroblasts (Figure $3 \mathrm{G}$ ), whereas the activities of the other complexes, including those containing mtDNA encoded subunits, were normal. These results indicate again the specificity of the NUBPL defect on CI function, and suggest that the mild reduction in mtDNA translation is unlikely to affect the function of the remaining complexes.

\section{Functional complementation assay}

To further confirm the pathogenicity of the pathogenic variants, we performed complementation experiments by overexpressing NUBPL wild-type cDNA in patient's and control fibroblasts by stable lentivirus transduction. The overexpression of the native protein fully restored the steady-state whole CI amount, assembly and enzyme activity and the correction of the mtDNA translation defect in the patient's cells (Figure 4A-F; Figure S6-7).

\section{Discussion}

NUBPL defect cause leukoencephalopathy with early onset $(0-24 \mathrm{~m})$ of developmental delay or motor regression and mild to severe ataxia, spasticity, dystonia, dysarthria and myopathy. Currently, 10 patients have been reported with variable disease course: four patients presented episodes of regression, two patients a progressive disease course; three had a stable disease course with acquisition of some milestones. Here, we describe for the first time a progressive multisystem clinical phenotype with type III renal tubular acidosis, osteoporosis, hepatomegaly, growth hormone deficiency, interstitial lung disease. Brain MRI confirmed the association of leukoencephalopathy with cerebellar, corpus callosum and deep cortical involvement. However, neurological features were not the most predominantly debilitating symptoms and they were including myopathy, hypotonia and neurocognitive deficit. NUBPL defect in our patient was due to compound heterozygous pathogenic variants: one very recently described variant in a different compound heterozygosity (c.311T $>$ C, p.Leu104Pro) (Balint et 
al., 2019) and a novel unreported variant (c.726C $>$ G, p.Phe242Leu). While the first pathogenic variant is localized in a Walker B motif essential for nucleotide bind, the second pathogenic variant is localized in proximity to the $\mathrm{CxxC}$ binding residues to the $\mathrm{Fe}-\mathrm{S}$ clusters. Therefore, we can hypothesize a genotype-phenotype correlation with the c.726C $>$ G, p.Phe242Le pathogenic variant responsible of the most severe and complex phenotype of our patient. Further studies with additional identified patients are needed to confirmed our hypothesize. NUBPL has been identified as a CI assembly factor, involved in the insertion of 4Fe-4S clusters in the CI N module (Sheftel et al., 2009). and in two 4Fe-S metallated subunits of the Q module, NDUFS2 and NDUFS3 (Sanchez-Caballero, Guerrero-Castillo, \& Nijtmans, 2016; Sheftel et al., 2009). We have performed an extensive functional analysis of our patient fibroblast cell line and we have demonstrated that NUBPL defect compromises the assembly of the whole CI. The assembly pathway of CI has recently been described as a modular process, which requires the synthesis of six independent multiprotein sub-modules $\left(\mathrm{Q}, \mathrm{P}_{\mathrm{P}-\mathrm{a}}, \mathrm{P}_{\mathrm{P}}-\mathrm{b}, \mathrm{P}_{\mathrm{D}}-\mathrm{a}, \mathrm{P}_{\mathrm{D}}-\mathrm{b}\right.$, $\mathrm{N}$ ), subsequently integrated to form the holoenzyme via a stepwise process involving at least 13 assembly factors and chaperons (Guerrero-Castillo et al., 2017). Both the $\mathrm{N}$ and the Q modules are pre-assembled before their incorporation in the holoenzyme. However, whilst the $\mathrm{Q}$ module is added to the membrane arm at an early stage, the insertion of the $\mathrm{N}$ module is one of the final steps of the complete assembly of CI (Guerrero-Castillo et al., 2017; SanchezCaballero et al., 2016). Previous structural analysis of CI by blue native gel electrophoresis in patients' cells with pathogenic variants in $\mathrm{N}$ and $\mathrm{Q}$ module subunits has shown the accumulation of stable $\mathrm{CI}$ intermediates corresponding to the unconnected $\mathrm{N}$ module and to the incomplete membrane arm, which can contain also Q module subunits, such as NDUFA9 and NDUFS2 (Lazarou, McKenzie, Ohtake, Thorburn, \& Ryan, 2007). Therefore, pathogenic variants causing the destabilization of either the $\mathrm{N}$ or the $\mathrm{Q}$ modules are not sufficient to determine the complete loss of the enzyme. Contrariwise, our results demonstrate a drastic effect on CI impaired NUBPL activity, leading to a decrease of CI subunits belonging to all three modules and a general loss of the fully assembled complex. This observation suggests that NUBPL is involved in the formation of both the peripheral and membrane arms of the enzyme. The analysis of mitochondrial complexes extracted with digitonin in 1D and 2D blue native gels allowed us to investigate the formation and the stability of supercomplexes in our patient cell line. As expected, in the absence of CI there is a redistribution of the remaining complexes, which leads to an increase in $\mathrm{CIII}_{2}$ and $\mathrm{CIII}_{2}+\mathrm{CIV}$ species, while monomer CIV does not seem to be affected. Interestingly, NUBPL defective cell line showed also reduced stability or impaired assembly of CIII. The formation of SC seems to be important for the structural stabilization of the individual enzymes (Moreno-Lastres et al., 2012). A current standard model suggests that partially assembled $\mathrm{CIII}_{2}$ is incorporated in $\mathrm{CI}+\mathrm{CIII} 2+\mathrm{CIV} \mathrm{SC}$, before the insertion of the Rieske protein (UQCRFS1), which is preferentially localized in SC rather than in free $\mathrm{CIII}_{2}$ [30]. This hypothesis is supported by the partial de-stabilization and degradation of $\mathrm{CIII}_{2}$ in our mutant cells, shown by $2 \mathrm{D} \mathrm{BN}$ WB.

We also observed a moderate defect in mitochondrial protein synthesis rate in the patient cells analyzed by $\left[{ }_{35} \mathrm{~S}\right]$-methionine labelling. This mildly affects all the mitochondrial encoded proteins, partially supporting a previous hypothesis on a role of NUBPL in mitochondrial translation (Wydro et al., 2013). The defect in the mitochondrial translation can be also responsible of instability of Complex III.

In conclusion, our results confirm the role of NUBPL in the assembly of CI and suggest an involvement of the protein in the stability of the whole enzyme. Further investigation is warranted to establish as whether this effect is due to a direct function of NUBPL on assembly of holo-CI, or to a severely disruptive structural consequence of impaired CI Fe-S metallation.

\section{Methods}




\section{Editorial Policies and Ethical Considerations}

This study was approved by the ethics committee of the Childern's Hospital Giannina Gaslini Institute, Genova (Italy) and it has been carried out in accordance with The Code of Ethics of the World Medical Association (Declaration of Helsinki). The patient's parents signed an informed consent approved by the Ethics Committee of the Gaslini Institute (available upon request), on the treatment of the biological material obtained from the patient.

None of the Authors has conflicts of interest concerning the present work.

\section{Cell Biology}

Human fibroblasts were grown in Dulbecco's Modified Eagle Medium (DMEM, Gibco \#10569010), supplemented with 10\% foetal bovine serum (Gibco \#10270-106), 1\% penicillin and streptomycin (Gibco \#15070-063) and $50 \mu \mathrm{g} / \mathrm{ml}$ uridine. Fibroblasts were immortalised by lentiviral transduction using the pLOX-Ttag-iresTK vector (Tronolab, Addgene \#12246).

Cells were lysed in TG lysis buffer $(20 \mathrm{mM}$ Tris- $\mathrm{HCl} \mathrm{pH}$ 7.5, $500 \mathrm{mM} \mathrm{NaCl}, 1 \mathrm{mM}$ EDTA, 1\% Triton-X-100, 10\% Glycerol, $1.5 \mathrm{mM} \mathrm{MgCl}$ ) and 1x protease inhibitor cocktail (Roche $\# 05056489001)$. The protein amount was detected with DC protein assay kit (BioRad \#5000111) and $20 \mu \mathrm{g}$ of protein were loaded in SDS-PAGE Nu-PAGE 4-12\% Bis-Tris gel (Invitrogen \#NP0321). Proteins were transferred to a PVDF membrane (Immobilon-P \#IPVH00010) at $100 \mathrm{~V}$ for 1 hour at $4^{\circ} \mathrm{C}$ in Tris-Glycine transfer buffer, $20 \%$ methanol, $0.25 \%$ SDS.

\section{Biochemistry}

Samples for Blue Native Gel Electrophoresis (BNGE) were prepared as described previously (Nijtmans, Henderson, \& Holt, 2002; Wittig, Braun, \& Schagger, 2006) with DDM or Digitonin in order to analyse the supercomplexes. Native samples were run through pre-cast NativePAGE 3\%-12\% Bis-Tris gels, while Novex NuPAGE 4\%-12\% Bis-Tris Gels (Life Technologies) were used for denaturing conditions. Proteins were blotted to a PVDF membrane (Immobilon-P \#IPVH00010) at $300 \mathrm{~mA}$ for 1.5 hours in bicarbonate transfer buffer $(0.318 \mathrm{mg} / \mathrm{ml} \mathrm{Na} 2 \mathrm{CO} 3,0.84 \mathrm{mg} / \mathrm{ml} \mathrm{NaHCO} 3)$. Samples were immunodetected using commercial specific antibodies. A detailed list can be found in Supp. Table 1.

In order to analyse the mitochondrial respiraotyr chain activities, cells samples were snapfrozen in liquid nitrogen and homogenized in $10 \mathrm{mM}$ phosphate buffer $(\mathrm{pH}$ 7.4). The spectrophotometric activity of CI, CII, CIII, CIV, and CS, was measured as described in Bugiani et al. (Bugiani et al., 2004).

$\mathrm{CI}$ activity was measured by following the decrease of NADH absorbance at $340 \mathrm{~nm}$. Proteins were incubated during 2 minutes at $30^{\circ} \mathrm{C}$ in a reaction mix containing $20 \mathrm{mM} \mathrm{KP}$ buffer $\mathrm{pH} 8$, $0.2 \mathrm{mM}$ NADH, $1 \mathrm{mM}$ sodium azide, $0.1 \% \mathrm{BSA}$ in $1 \mathrm{mM}$ EDTA $\mathrm{pH}=7.4$. The reaction was initiated adding $50 \mu \mathrm{M} \mathrm{CoQ}$, followed for $2 \mathrm{~min}$, and then inhibited with $5 \mu \mathrm{M}$ rotenone. CII activity was performed at $30^{\circ} \mathrm{C}$ pre incubating proteins with $50 \mathrm{mM} \mathrm{KP}$ Buffer $\mathrm{pH}=7.0,1.5$ $\mathrm{mM} \mathrm{KCN}$ and $0.1 \mathrm{mM}$ DCPIP. This reaction was followed after the injection of $16 \mathrm{mM}$ succinate (SDH activity) and $50 \mathrm{mM} \mathrm{CoQ} \mathrm{(CII} \mathrm{activity)} \mathrm{at} 600 \mathrm{~nm}$ for two minutes. Complex III was assessed measuring the reduction of cytochrome $\mathrm{c}$ at $550 \mathrm{~nm}$ for $2 \mathrm{~min}$ at $30^{\circ} \mathrm{C}$. Proteins were incubated with $50 \mathrm{mM} \mathrm{KP}$ buffer $\mathrm{pH}=7.4,2 \mathrm{mM} \mathrm{NaN3}, 1 \mathrm{mg} / \mathrm{ml}$ BSA (in EDTA $10 \mathrm{mM}$ $\mathrm{pH}=7.4), 50 \mathrm{mM}$ Cytochrome c (SIGMA \#C7752), and $50 \mathrm{mM}$ reduced decylubiquinone (DBH2, Sigma \#D7911). CIV activity was measured at $550 \mathrm{~nm}$ following cytochrome c oxidation for two minutes at $37^{\circ} \mathrm{C}$. Assay was performed in $90-95 \%$ reduced cytochrome c $(1.3 \mathrm{mg} / \mathrm{ml}$, SIGMA \#C7752) in $50 \mathrm{mM} \mathrm{KP}$ buffer $\mathrm{pH}=7.0$. All enzymatic activities were normalized for protein quantification (DC assay kit, Bio-Rad) and for citrate synthase (CS) activity. For CS activity measurements, proteins were incubated at $30^{\circ} \mathrm{C}$ with $75 \mathrm{mM}$ Tris- $\mathrm{HCl}$ Buffer $\mathrm{pH}=8.0,0.1 \mathrm{mM} \mathrm{5,5^{ \prime }}$ - Dithiobis-(2-nitrobenzoic acid) (DTNB) and 0.4 mM Acetyl- 
CoA. The reaction was initiated adding $0.5 \mathrm{mM}$ Oxalacetate and followed at $412 \mathrm{~nm}$ for two minutes.

To analyse translation of mitochondrially encoded proteins metabolic labelling was performed as previously described (Rorbach et al., 2014). Cells were grown as described and, then the standard medium was replaced with methionine/cysteine free DMEM (Sigma-Aldrich) supplemented with $2 \mathrm{mM}$ l-glutamine, $48 \mu \mathrm{g} / \mathrm{ml}$ cysteine, and $50 \mu \mathrm{g} / \mathrm{ml}$ uridine. The cells were incubated for $2 \AA \sim 10 \mathrm{~min}$ in this medium and then transferred to methionine/cysteine-free DMEM containing 10\% ( $\mathrm{vol} / \mathrm{vol})$ dialyzed FCS and emetine dihydrochloride $(100 \mu \mathrm{g} / \mathrm{ml})$ to inhibit cytosolic translation. Cells were incubated for $10 \mathrm{~min}$ before addition of $120 \mu \mathrm{Ci} / \mathrm{ml}$ of [35S]methionine. Labeling was performed for $60 \mathrm{~min}$, and then the cells were washed twice with standard growth medium. Protein samples $(30 \mu \mathrm{g})$ were separated on 10-20\% SDS-PAGE gels, and products were visualized and quantified using a PhosphorImager system with ImageQuant software (Molecular Dynamics, GE Healthcare).

\section{Statistics}

Statistical analyses were performed using GraphPad Prism software version XXX. P values less than 0.05 according to a two-tailed Student's t-test were considered significant.

\section{Acknowledgments}

We thank Dr. Erika Fernandez-Vizarra for her help and advice. Our work was supported by the Core Grant from the MRC (Grant MC_UU_00015/5); ERC Advanced under Grant FP7322424 and NRJ-Institut de France Grant (to M.Z.); European Commission under 'Marie Skłodowska-Curie Actions', Individual Fellowship - Reintegration Panel (Mitobiopath705560)(CG).

\section{References}

Balint, B., Charlesworth, G., Stamelou, M., Carr, L., Mencacci, N. E., Wood, N. W., \& Bhatia, K. P. (2019). Mitochondrial complex I NUBPL mutations cause combined dystonia with bilateral striatal necrosis and cerebellar atrophy. Eur J Neurol, 26(9), 1240-1243. doi:10.1111/ene.13956

Birrell, J. A., Morina, K., Bridges, H. R., Friedrich, T., \& Hirst, J. (2013). Investigating the function of [2Fe-2S] cluster N1a, the off-pathway cluster in complex I, by manipulating its reduction potential. Biochem J, 456(1), 139-146. doi:10.1042/BJ20130606

Bugiani, M., Invernizzi, F., Alberio, S., Briem, E., Lamantea, E., Carrara, F., . . Zeviani, M. (2004). Clinical and molecular findings in children with complex I deficiency. Biochim Biophys Acta, 1659(2-3), 136-147. doi:10.1016/j.bbabio.2004.09.006

Bych, K., Kerscher, S., Netz, D. J., Pierik, A. J., Zwicker, K., Huynen, M. A., . . . Balk, J. (2008). The iron-sulphur protein Ind1 is required for effective complex I assembly. EMBO J, 27(12), 1736-1746. doi:10.1038/emboj.2008.98

Calvo, S. E., Tucker, E. J., Compton, A. G., Kirby, D. M., Crawford, G., Burtt, N. P., . . . Mootha, V. K. (2010). High-throughput, pooled sequencing identifies mutations in NUBPL and FOXRED1 in human complex I deficiency. Nat Genet, 42(10), 851-858. doi:10.1038/ng.659

Garone, C., Donati, M. A., Sacchini, M., Garcia-Diaz, B., Bruno, C., Calvo, S., . . . Dimauro, S. (2013). Mitochondrial encephalomyopathy due to a novel mutation in ACAD9. JAMA Neurol, 70(9), 1177-1179. doi:10.1001/jamaneurol.2013.3197

Guerrero-Castillo, S., Baertling, F., Kownatzki, D., Wessels, H. J., Arnold, S., Brandt, U., \& Nijtmans, L. (2017). The Assembly Pathway of Mitochondrial Respiratory Chain Complex I. Cell Metab, 25(1), 128-139. doi:10.1016/j.cmet.2016.09.002 
Kevelam, S. H., Rodenburg, R. J., Wolf, N. I., Ferreira, P., Lunsing, R. J., Nijtmans, L. G., . . . van der Knaap, M. S. (2013). NUBPL mutations in patients with complex I deficiency and a distinct MRI pattern. Neurology, 80(17), 1577-1583. doi:10.1212/WNL.0b013e31828f1914

Lazarou, M., McKenzie, M., Ohtake, A., Thorburn, D. R., \& Ryan, M. T. (2007). Analysis of the assembly profiles for mitochondrial- and nuclear-DNA-encoded subunits into complex I. Mol Cell Biol, 27(12), 4228-4237. doi:10.1128/MCB.00074-07

Leipe, D. D., Koonin, E. V., \& Aravind, L. (2003). Evolution and classification of P-loop kinases and related proteins. J Mol Biol, 333(4), 781-815. Retrieved from https://www.ncbi.nlm.nih.gov/pubmed/14568537

Mimaki, M., Wang, X., McKenzie, M., Thorburn, D. R., \& Ryan, M. T. (2012). Understanding mitochondrial complex I assembly in health and disease. Biochim Biophys Acta, 1817(6), 851-862. doi:10.1016/j.bbabio.2011.08.010

Moreno-Lastres, D., Fontanesi, F., Garcia-Consuegra, I., Martin, M. A., Arenas, J., Barrientos, A., \& Ugalde, C. (2012). Mitochondrial complex I plays an essential role in human respirasome assembly. Cell Metab, 15(3), 324-335. doi:10.1016/j.cmet.2012.01.015

Netz, D. J., Pierik, A. J., Stumpfig, M., Bill, E., Sharma, A. K., Pallesen, L. J., . . Lill, R. (2012). A bridging [4Fe-4S] cluster and nucleotide binding are essential for function of the Cfd1-Nbp35 complex as a scaffold in iron-sulfur protein maturation. J Biol Chem, 287(15), 12365-12378. doi:10.1074/jbc.M111.328914

Nijtmans, L. G., Henderson, N. S., \& Holt, I. J. (2002). Blue Native electrophoresis to study mitochondrial and other protein complexes. Methods, 26(4), 327-334. doi:10.1016/S1046-2023(02)00038-5

Nouws, J., Nijtmans, L. G., Smeitink, J. A., \& Vogel, R. O. (2012). Assembly factors as a new class of disease genes for mitochondrial complex I deficiency: cause, pathology and treatment options. Brain, 135(Pt 1), 12-22. doi:10.1093/brain/awr261

Rorbach, J., Boesch, P., Gammage, P. A., Nicholls, T. J., Pearce, S. F., Patel, D., . . Minczuk, M. (2014). MRM2 and MRM3 are involved in biogenesis of the large subunit of the mitochondrial ribosome. Mol Biol Cell, 25(17), 2542-2555. doi:10.1091/mbc.E14-010014

Sanchez-Caballero, L., Guerrero-Castillo, S., \& Nijtmans, L. (2016). Unraveling the complexity of mitochondrial complex I assembly: A dynamic process. Biochim Biophys Acta, 1857(7), 980-990. doi:10.1016/j.bbabio.2016.03.031

Sheftel, A. D., Stehling, O., Pierik, A. J., Netz, D. J., Kerscher, S., Elsasser, H. P., . . Lill, R. (2009). Human ind1, an iron-sulfur cluster assembly factor for respiratory complex I. Mol Cell Biol, 29(22), 6059-6073. doi:10.1128/MCB.00817-09

Signes, A., \& Fernandez-Vizarra, E. (2018). Assembly of mammalian oxidative phosphorylation complexes I-V and supercomplexes. Essays Biochem, 62(3), 255270. doi:10.1042/EBC20170098

Tenisch, E. V., Lebre, A. S., Grevent, D., de Lonlay, P., Rio, M., Zilbovicius, M., . . Boddaert, N. (2012). Massive and exclusive pontocerebellar damage in mitochondrial disease and NUBPL mutations. Neurology, 79(4), 391. doi:10.1212/WNL.0b013e3182611232

Tucker, E. J., Mimaki, M., Compton, A. G., McKenzie, M., Ryan, M. T., \& Thorburn, D. R. (2012). Next-generation sequencing in molecular diagnosis: NUBPL mutations highlight the challenges of variant detection and interpretation. Hum Mutat, 33(2), 411418. doi:10.1002/humu.21654

Vinothkumar, K. R., Zhu, J., \& Hirst, J. (2014). Architecture of mammalian respiratory complex I. Nature, 515(7525), 80-84. doi:10.1038/nature13686 
Wittig, I., Braun, H. P., \& Schagger, H. (2006). Blue native PAGE. Nat Protoc, 1(1), 418-428. doi:10.1038/nprot.2006.62

Wydro, M. M., Sharma, P., Foster, J. M., Bych, K., Meyer, E. H., \& Balk, J. (2013). The evolutionarily conserved iron-sulfur protein INDH is required for complex I assembly and mitochondrial translation in Arabidopsis [corrected]. Plant Cell, 25(10), 40144027. doi:10.1105/tpc.113.117283 

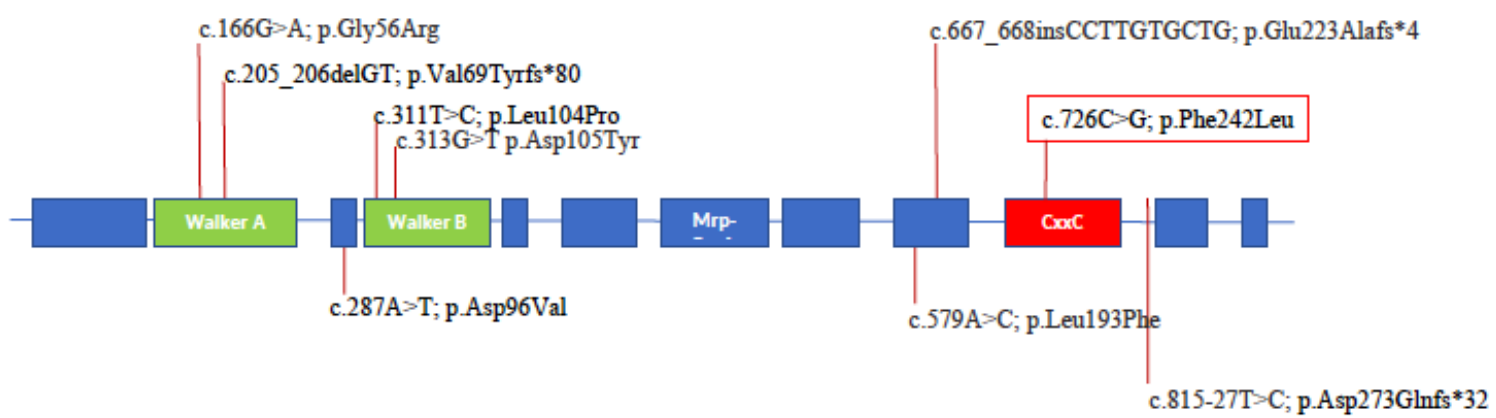

Figure 1 - Schematic representation of NUBPL exons and reported pathogenic variants. The red box highlights the novel pathogenic variant described in the current report. 


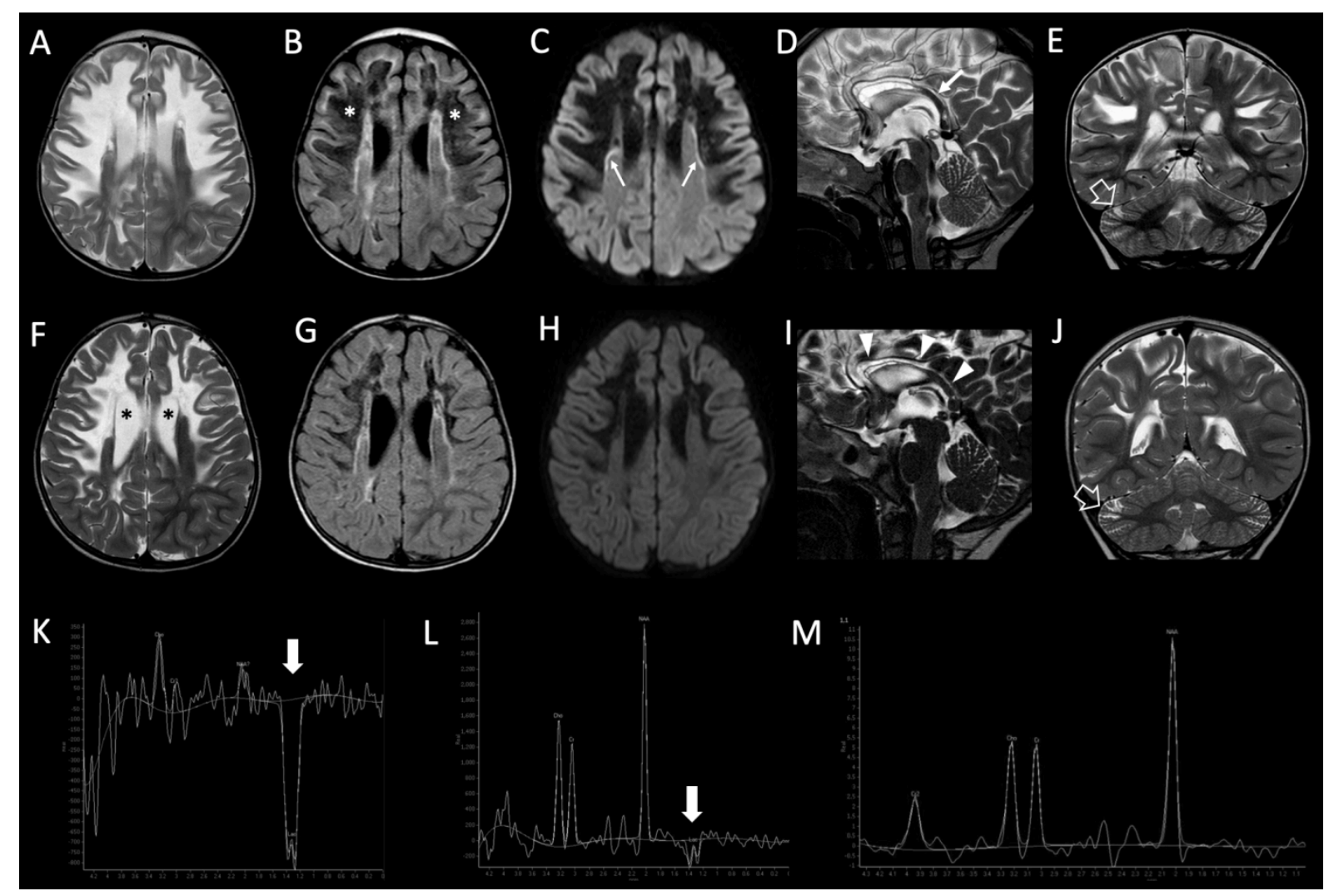

Figure 2. Brain MRI and MR Spectroscopy findings of the patient obtained at 2.5 years (A-E, $\mathrm{K}, \mathrm{L})$ and 7 years of age (F-J, M). Axial T2-weighted (A, F), fluid-attenuated inversion recovery (FLAIR) $(B, G)$, diffusion-weighted images (DWI) $(C, H)$, sagittal T2-weighted (D, I) and coronal T2-weighted (E, J) images are shown. The initial MRI shows diffuse cerebral white matter abnormalities, mainly in the frontal and right parietal lobes (A, B), involving both the periventricular and subcortical regions, with central rarefaction (asterisks, B). The abnormal cerebral white matter is surrounded by a rim of abnormal solid tissue, with linear areas of restricted diffusion (arrows, C). There is swelling and T2-hyperintensities at the level of the anterior portions of the corpus callosum and splenium, with preservation of the fibres connecting the pericentral cortex (arrow, D). There is a very small area of signal abnormality of the cerebellar cortex in the right hemisphere (empty arrow, E). The late MRI reveals improvement of the deep white matter changes with absence of restricted diffusion $(\mathrm{G}, \mathrm{H})$, and thinning of the affected white matter with mild enlargement of the lateral ventricles (asterisks, F). There is atrophy of the corpus callosum (arrowheads, I). The cerebellar abnormalities slightly worsen (empty arrow, J). MR spectroscopy at 2.5 years of age demonstrate very high lactate peaks in the affected white matter (arrow, K) and smaller lactate peaks in the spared, normal-appearing white matter (arrow, L). Follow-up MR spectroscopy performed at 7 years of age reveals no significant lactate peaks in the affected cerebral white matter (M). 

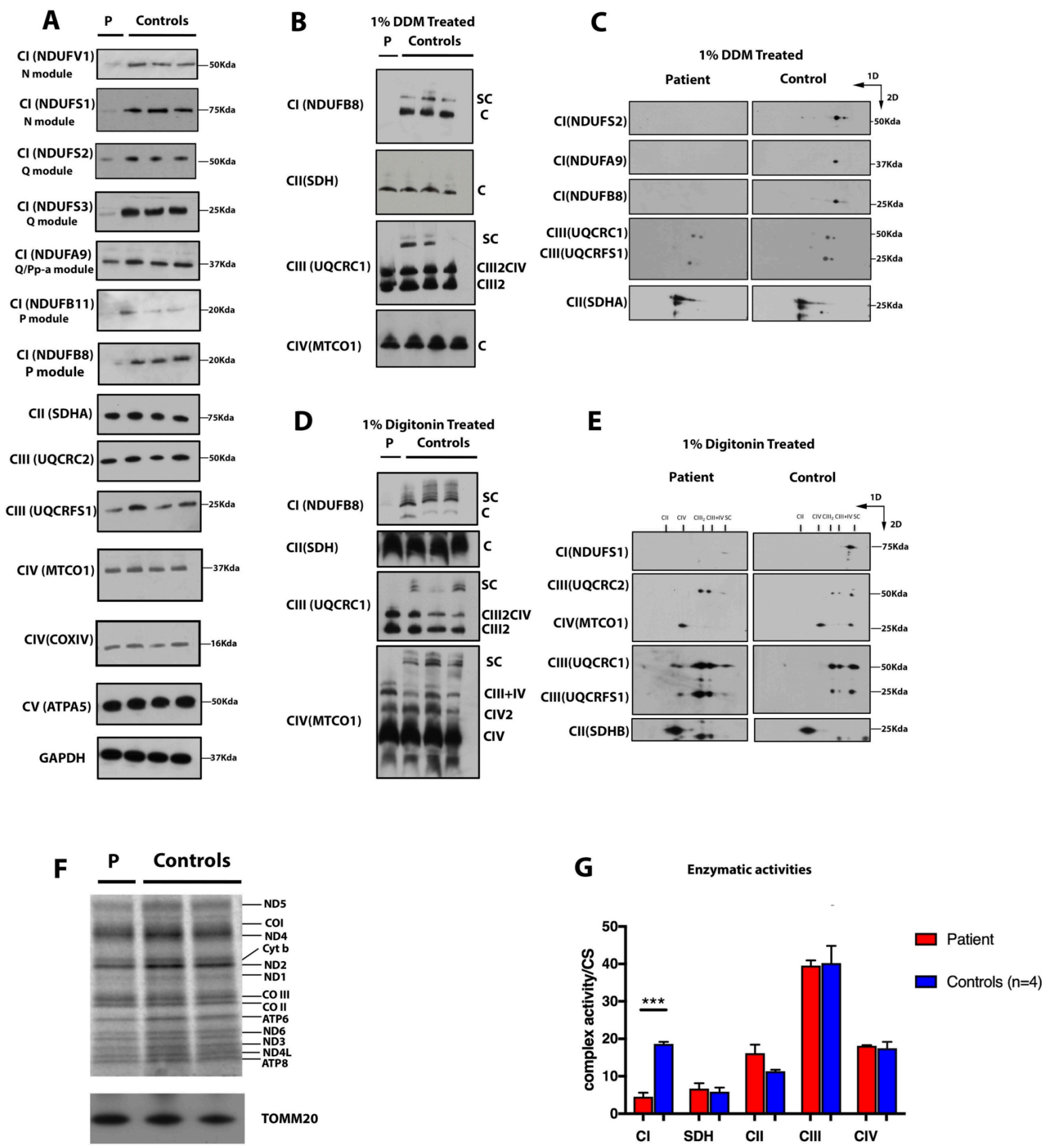

Figure 3 - Functional characterisation of NUBPL defective immortalised fibroblasts vs three independent controls: the analyses of A) steady-state level of CI (NDUFV1, NDUFS1, NDUFS2, NDUFS3, NDUFA9, NDUFB11, NDUFB8), CII (SDHA), CIII (UQCRC2, UQCRFS1), CIV (MTCO1, COXIV), CV (ATPA5) and loading control (GAPDH) by WB analysis of SDS page from fibroblast lysates; B) 1D BN-PAGE and C) 2D BN- PAGE WB study with $1 \%$ DDM treated proteins; D) $1 \mathrm{D}$ BN-PAGE and E) 2D BN- PAGE study with $1 \%$ Digitonin treated proteins; F) $35 \mathrm{~S}$-methionine labelling for mitochondrial protein translation were performed in patient's and controls immortalised fibroblasts; and, G) Spectrophotometer analysis of mitochondrial respiratory chain complex activities in fibroblast lysates. Data in figures are given as mean +/- SEM based on at least 3 biological replicates. 
$\mathrm{CI}=\mathrm{NADH}$ :ubiquinone oxidoreductase; $\mathrm{CII}=$ succinate:ubiquinone oxidoreductase ; $\mathrm{SDH}=$ Succinate dehydrogenase; $\mathrm{CIII}=$ quinol-cytochrome $\mathrm{c}$ reductase; $\mathrm{CIV}=$ cytochrome $\mathrm{c}$ oxidase; $\mathrm{CV}=\mathrm{F}_{0} \mathrm{~F}_{1}$-ATPase 

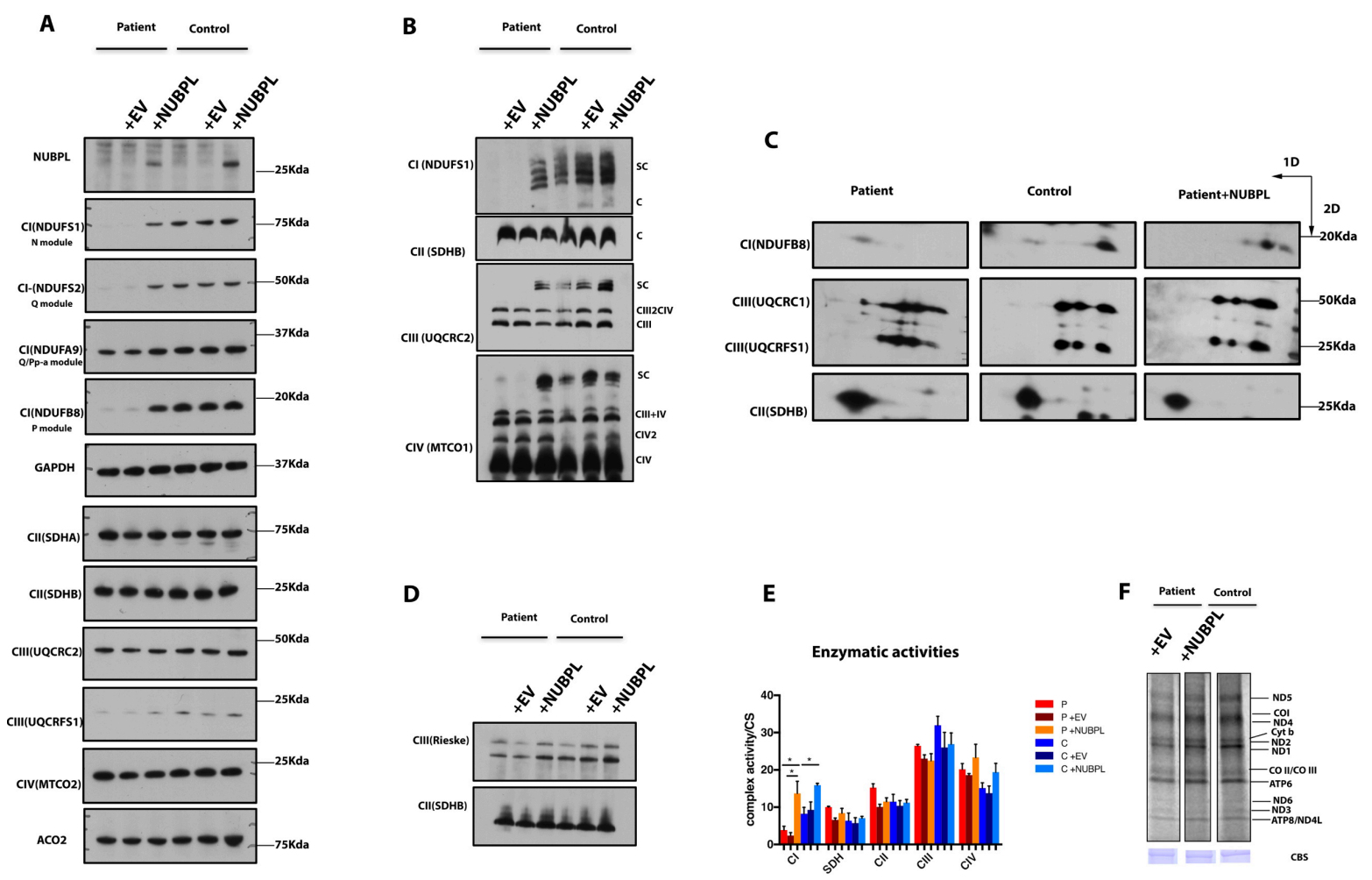

Figure 4 - Stable lentiviral transfection of wild-type NUBPL restored the defective Complex I as demonstrated by analysing the A) Steady state levels of NUBPL and CI subunits (NDUFS1, NDUFS2, NDUFA9, NDUFB8) by SDS page analysis; B) 1D and C) 2D BN PAGE WB of samples treated with 1\% DDM and D) 1D BN PAGE of Rieske protein in 1\%DDM treated samples; E) spectrophotometer analysis of mitochondrial respiratory chain activities; F) $35 \mathrm{~S}$-methionine labelling for mitochondrial proteins translation. Data in figures are given as mean $+/$ - SEM based on at least 3 biological replicates.

$\mathrm{CI}=\mathrm{NADH}$ :ubiquinone oxidoreductase; $\mathrm{CII}=$ succinate:ubiquinone oxidoreductase ; $\mathrm{SDH}=$ Succinate dehydrogenase; $\mathrm{CIII}=$ ubiquinol-cytochrome $\mathrm{c}$ reductase; $\mathrm{CIV}=$ cytochrome $\mathrm{c}$ oxidase. 


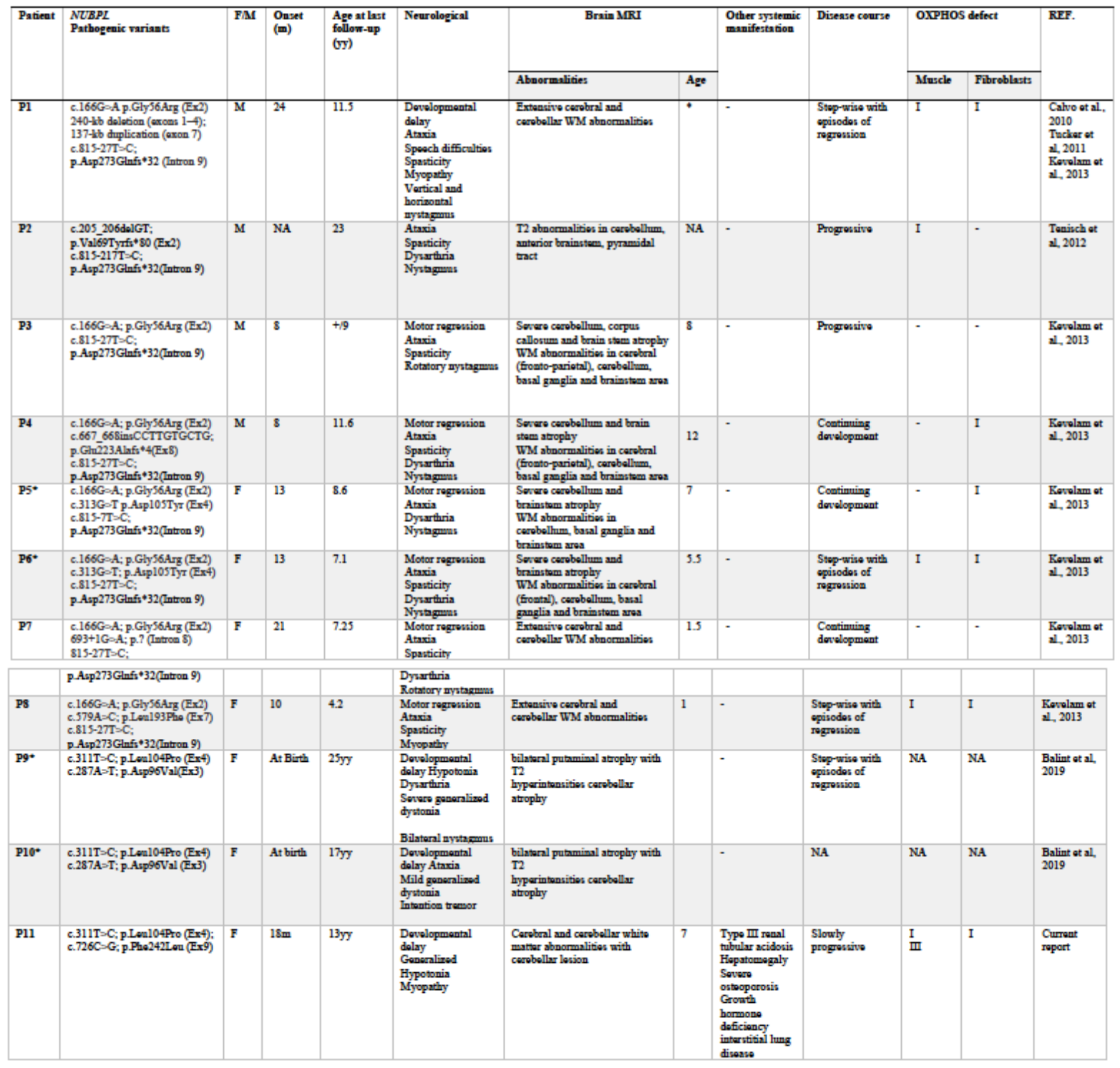

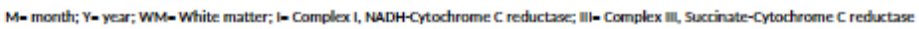

Table I - Clinical, molecular genetics and biochemical features of NUBPL patients 


\begin{tabular}{|l|c|c|}
\hline & Patient & Controls \\
\hline NADH DEHYDROGENASE & 20.1 & $28.67-39.53$ \\
\hline SUCCINATE DEHYDROGENASE & 0.96 & $0.49-0.73$ \\
\hline $\begin{array}{l}\text { NADH-CYTOCHROME C REDUCTASE } \\
\text { (COMPLEXES I+III) }\end{array}$ & 0.10 & $0.66-1.52$ \\
\hline $\begin{array}{l}\text { SUCCINATE-CITOCHROME C REDUCTASE } \\
\text { (COMPLEXES II+III) }\end{array}$ & 0.46 & $0.46-0.88$ \\
\hline CYTOCHROME C OXIDASE & 3.92 & $1.78-2.46$ \\
\hline CITRATE SINTHASE & 21.3 & $7.82-10.90$ \\
\hline
\end{tabular}

Table II: mitochondrial respiratory chain activities $(\mu \mathrm{mole} / \mathrm{min} / \mathrm{g})$ in muscle homogenate 


\section{Supplemental material}

Supplemental Figure 1 - ClustalW alignment of human NUBPL (NP_079428.2) and cytosolic human orthologues of cytosolic Fe/S proteins Cfd1/Nbp35 NUBP1 (NP_002475.2).

The main domains are indicated in yellow, while in green are highlighted the mutations found in the patient.

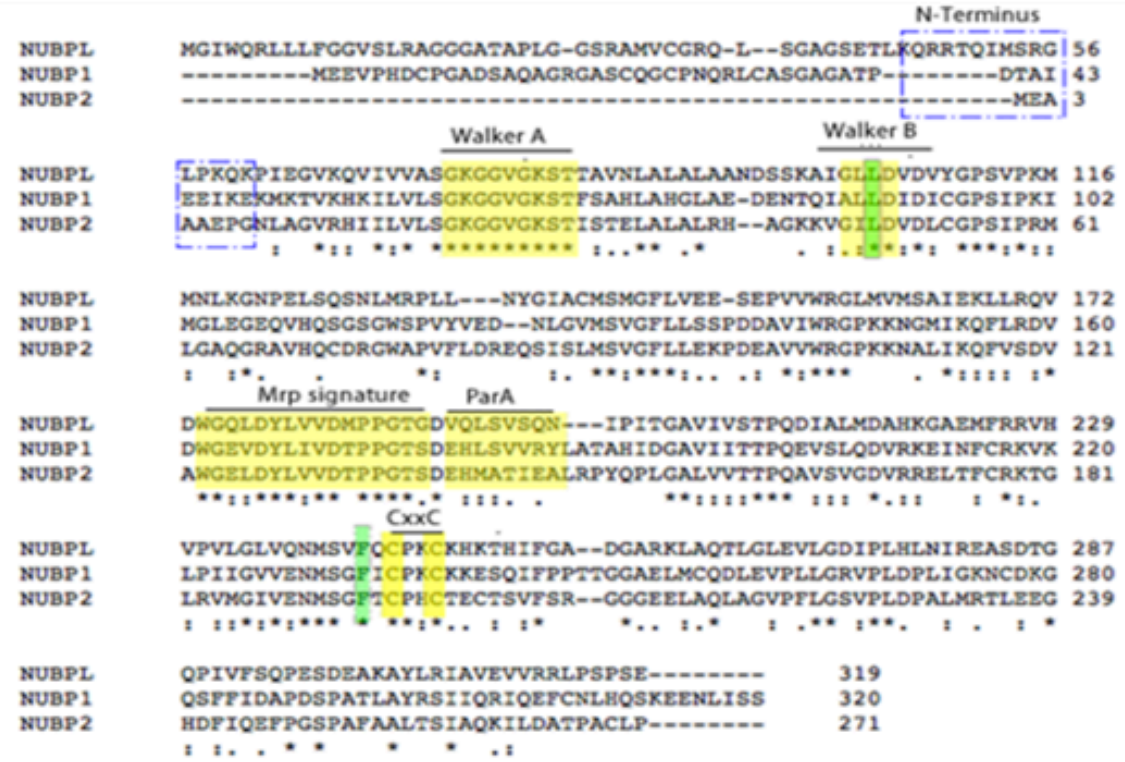




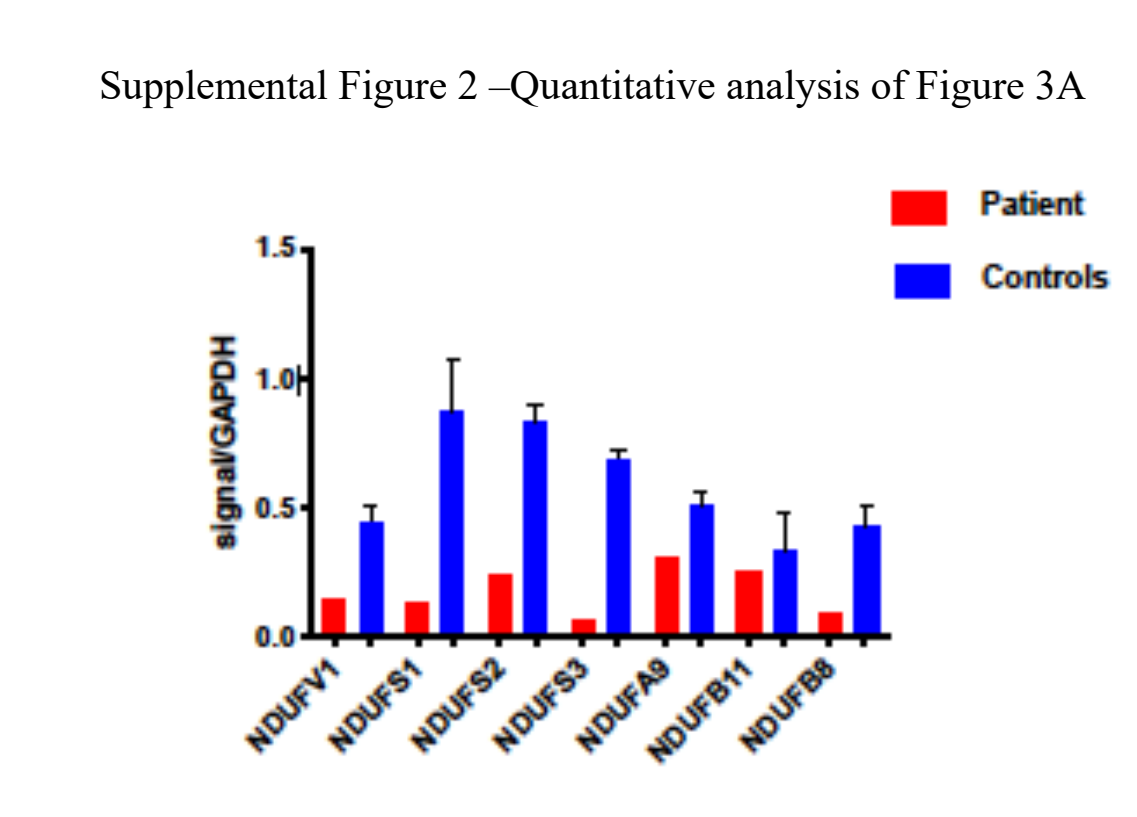


Supplemental Figure 3 - Digitonin treated protein from ACAD9 defective human fibroblast cell line and control: A) 1D BN Page showing reduction of Complex I in ACAD9 cell line; B) $2 \mathrm{D}$

BN-Page showing a very mild accumulation of sub-assemblies in ACAD9 cell line.

A

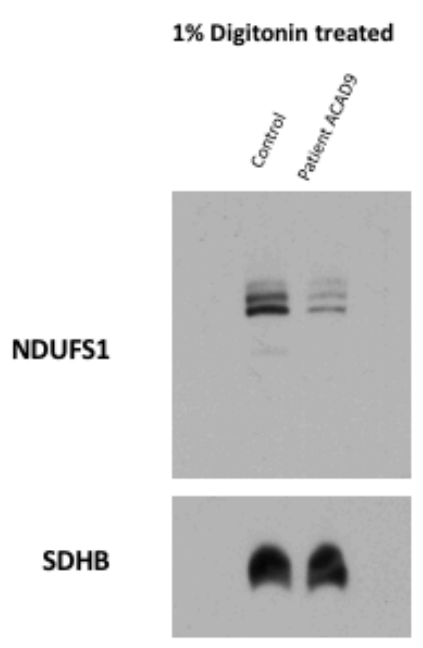

B

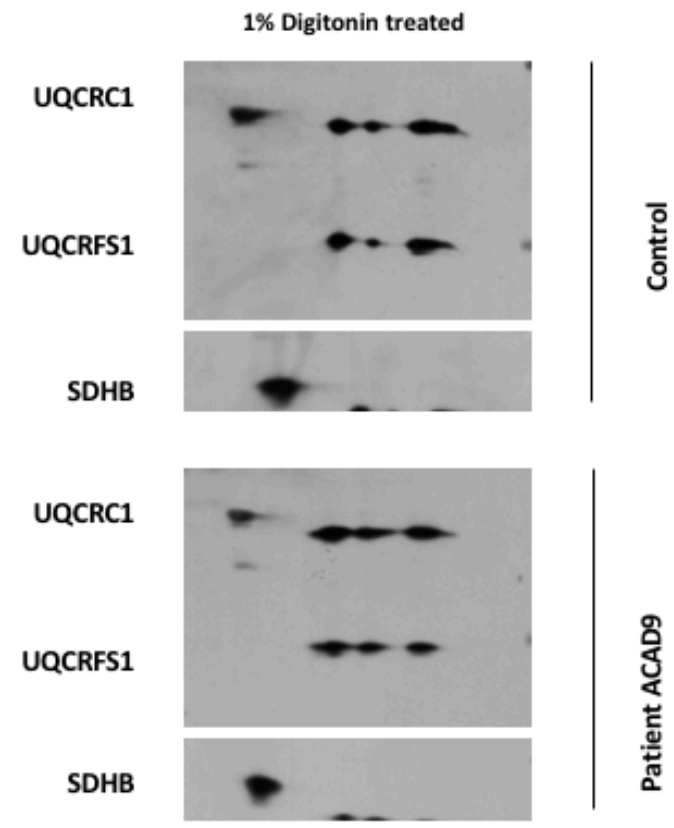


Supplemental Figure 4- Quantitative analysis of Figure 3F

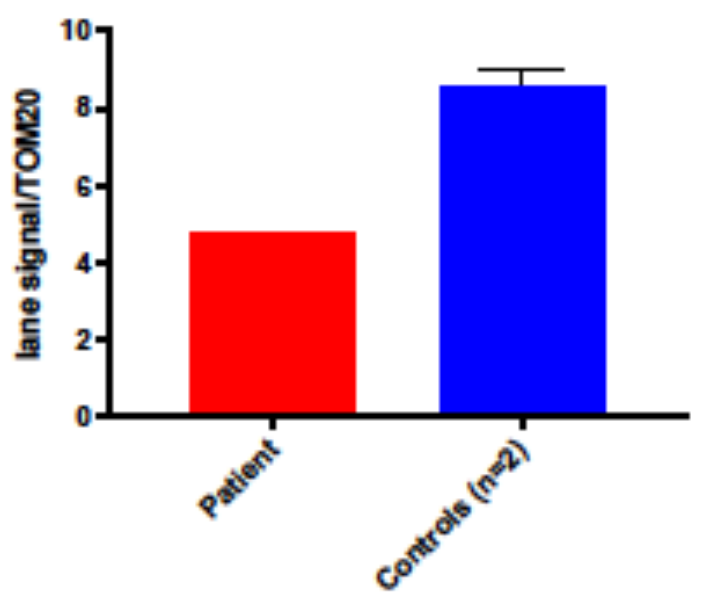


Supplemental Figure 5 - 35-S-methionine labelling for mitochondrial proteins translation in ACAD9 defective human fibroblast cell line versus control

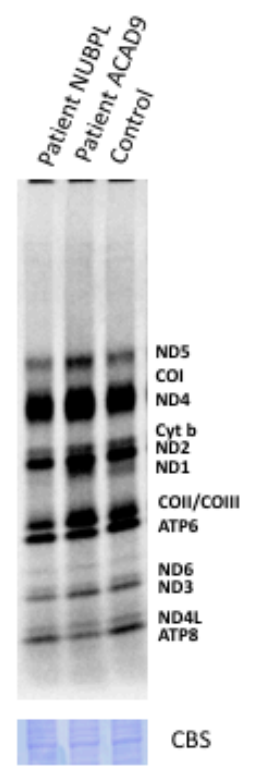


Supplemental Figure 6 - Quantitative analysis of Figure 4A

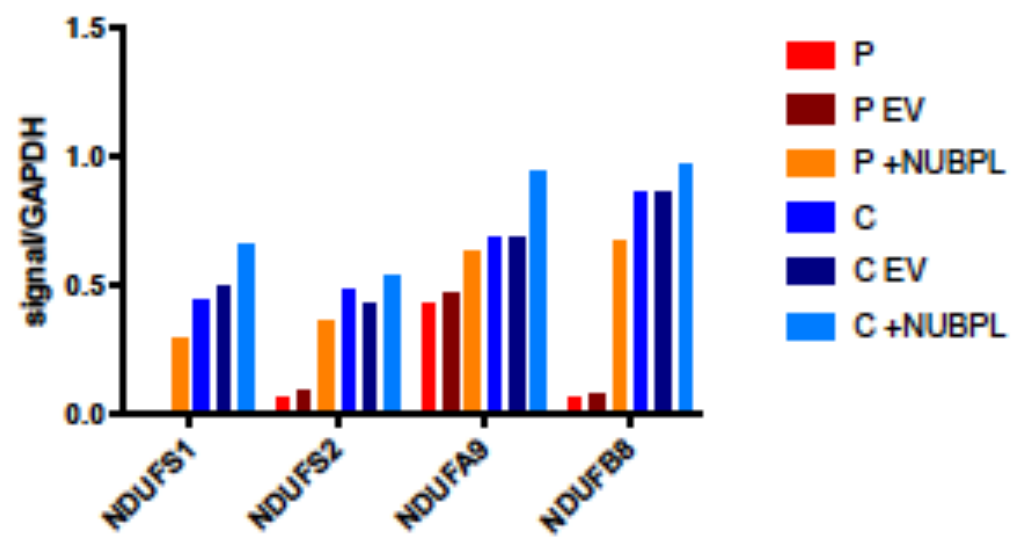


Supplemental Figure 7 - Quantitative analysis of Figure 4F

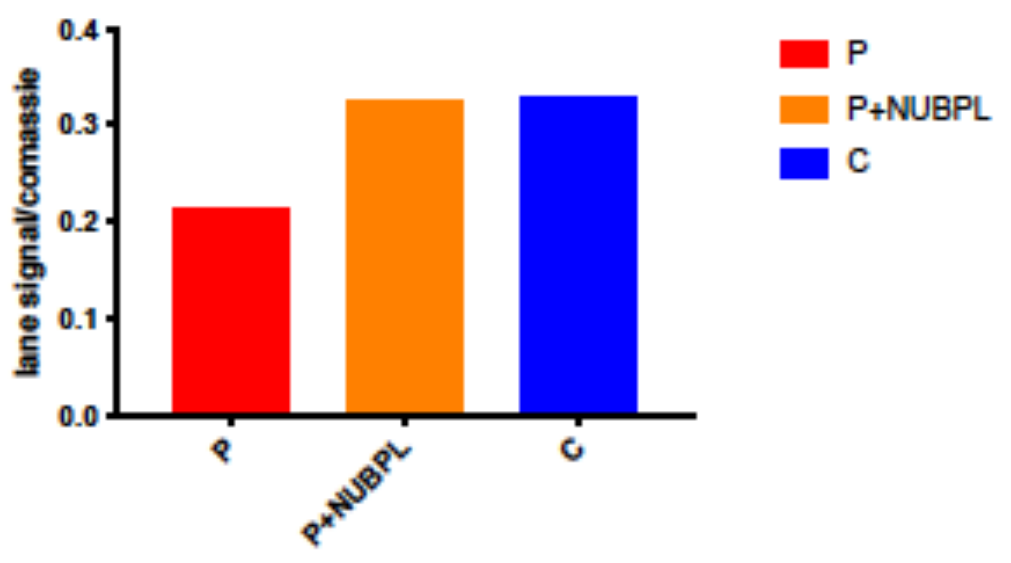


Supplemental Table 1- List of antibodies

\begin{tabular}{|c|c|c|c|c|c|}
\hline Target & Type & Producer & $\begin{array}{l}\text { Catalogue } \\
\text { Number }\end{array}$ & Dilution & Complex \\
\hline NDUFVl & $\begin{array}{l}\text { Mous } \\
\mathrm{e}\end{array}$ & $\begin{array}{l}\text { Santa Cruz } \\
\text { Biotechnology }\end{array}$ & sc-100566 & $1: 100$ & $\mathrm{CI}$ - $\mathrm{N}$ module \\
\hline NDUFS1 & & ABCAM & ab169540 & $1: 1000$ & $\mathrm{CI}$ - $\mathrm{N}$ module \\
\hline NDUFS2 & rabbit & ABCAM & ab110249 & $1: 5000$ & $\mathrm{CI}$ - $\mathrm{N}$ module \\
\hline NDUFS3 & rabbit & ABCAM & ab110246 & $1: 500$ & CI - Q module \\
\hline NDUFA9 & $\begin{array}{l}\text { mouse } \\
\text { mouse }\end{array}$ & $\begin{array}{l}\text { LifeTechnologi } \\
\text { es }\end{array}$ & LS459100 & $1: 1000$ & $\mathrm{CI}-\mathrm{Q}$ module \\
\hline NDUFB11 & & Proteintech & 16720-1-AP & $1: 500$ & $\mathrm{CI}$ - $\mathrm{P}_{\mathrm{D}-\mathrm{a}}$ module \\
\hline & mouse & ProteinTech & & & \\
\hline & & Europe & & & \\
\hline NDUFB8 & mouse & ABCAM & ab110242 & $1: 500$ & $\mathrm{CI}-\mathrm{P}_{\mathrm{p}^{-b} \mathrm{~b}}$ module \\
\hline SDHA & mouse & ABCAM & ab14715 & $1: 1000$ & $\mathrm{CII}$ - loading \\
\hline SDHB & mouse & ABCAM & ab14714 & $1: 1000$ & CII-loading \\
\hline UQCRCl & mouse & ABCAM & ab110252 & $1: 1000$ & CIII \\
\hline UQCRC2 & mouse & ABCAM & ab14745 & $1: 1000$ & CIII \\
\hline UQCRFSI & mouse & ABCAM & ab14746 & $1: 1000$ & CIII \\
\hline MTCOl & mouse & ABCAM & ab14705 & $1: 1000$ & CIV \\
\hline MTCO2 & mouse & ABCAM & ab110258 & $1: 1000$ & CIV \\
\hline ATPA5 & mouse & ABCAM & ab14748 & $1: 1000$ & $\mathrm{CV}$ \\
\hline NUBPL & mouse & NovUS & NBP2-01360 & $1: 500$ & $\begin{array}{l}\text { CI- assembly } \\
\text { factor }\end{array}$ \\
\hline TOMM20 & rabbit & $\begin{array}{l}\text { Santa Cruz } \\
\text { Biotechnology }\end{array}$ & Sc-11415 & $1: 1000$ & NA \\
\hline $\begin{array}{l}\text { ACONITAS } \\
\text { E } 2\end{array}$ & mouse & ABCAM & ab110321 & $1: 1000$ & NA \\
\hline GAPDH & mouse & ABCAM & ab8245 & $1: 5000$ & NA \\
\hline
\end{tabular}




\begin{tabular}{llllll}
\hline Mouse IgG & Goat & PROMEGA & W402B & $1: 3000$ & $\begin{array}{l}\text { Secondary } \\
\text { Antibody }\end{array}$ \\
& & & & & Secondary \\
Rabbit IgG & \multirow{2}{*}{ Goat } & PROMEGA & W401B & $1: 3000$ & Antibody \\
\hline
\end{tabular}

$\mathrm{CI}=\mathrm{NADH}$ :ubiquinone oxidoreductase $\mathbf{C I I}=$ Succinate dehydrogenase $; \mathrm{CIII}=$ quinolcytochrome $c$ reductase; $\mathrm{CIV}=$ cytochrome $c$ oxidase; $\mathrm{CV}=\mathrm{F}_{0} \mathrm{~F}_{1}-\mathrm{ATPase} ; \mathrm{NA}=$ Not applicable 